\title{
Activity of the streptogramin antibiotic etamycin against methicillin-resistant Staphylococcus aureus
}

\author{
Nina M Haste ${ }^{1,2}$, Varahenage R Perera ${ }^{3}$, Katherine N Maloney ${ }^{2,4}$, Dan N Tran ${ }^{3}$, Paul Jensen², William Fenical ${ }^{2}$, \\ Victor Nizet $^{1,3}$ and Mary E Hensler ${ }^{3}$
}

The alarming rise of hospital- and community-associated methicillin-resistant Staphylococcus aureus (HA- and CA-MRSA) infections has prompted a desperate search for novel antibiotics. We discovered the streptogramin etamycin produced by an actinomycete species isolated from the coast of Fiji, the first time this antibiotic has been identified from a marine microbe. Etamycin was extracted and purified from this strain (CNS-575) and identified as a three-rotamer species by 2D NMR spectroscopy. Etamycin demonstrated potent activity against HA- and CA-MRSA in microbroth dilution assays, with minimum inhibitory concentrations (MIC) as low as $1-2 \mathrm{mg} \mathrm{I}^{-1}$ against HA- and CA-MRSA strains. Furthermore, etamycin was also active against other Gram-positive and several Gram-negative pathogens and was found to be non-cytotoxic at concentrations more than 20-fold above MIC. Etamycin displayed favorable time-kill kinetics compared with the first-line MRSA antibiotic, vancomycin, and also conferred significant protection from mortality in a murine model of systemic lethal MRSA infection. These data emphasize the utility of the marine environment as a relatively untapped source of antibiotics against major drug-resistant human pathogens. These studies will also guide future isolation and preclinical development of depsipeptide anti-MRSA compounds from marine-derived actinomycetes.

The Journal of Antibiotics (2010) 63, 219-224; doi:10.1038/ja.2010.22; published online 26 March 2010

Keywords: etamycin; marine-derived actinomycete; MRSA; streptogramin

\section{INTRODUCTION}

The alarming rise of methicillin-resistant Staphylococcus aureus (MRSA) infections in several countries worldwide has provided urgency to the search for structurally novel antibiotics that target these organisms. ${ }^{1}$ Hospital-associated (HA)-MRSA are frequently resistant to multiple antibiotic classes, and rapidly emergent strains of community-associated (CA)-MRSA, which have demonstrated unusually high virulence and ease of transmission, now represent the most common cause of skin and soft tissue infections in the United States. ${ }^{2,3}$ Furthermore, the approval of novel antibiotics for human medicine has not kept pace with the development of resistance by important bacterial pathogens such as MRSA. Just a few drugs with novel scaffolds (linezolid, daptomycin) have been introduced to the antibacterial formulary in the past 11 years, and only three new antibacterial compounds are in advanced stages of clinical development. ${ }^{4,5}$ Both HA- and CA-MRSA strains have already demonstrated the capacity to evolve resistance to linezolid and daptomycin.

Natural products represent a vast source of potential new chemical structures possessing antimicrobial activity. At least three of every four current antibacterial agents are related in some way to natural products, ${ }^{6}$ and a majority of these discoveries have come from filamentous bacteria of the order Actinomycetales (actinomycetes). More than 9000 biologically active molecules have been isolated from actinomycetes, yielding $>60$ pharmaceutical agents used in the medical or agricultural fields. ${ }^{6}$ Although terrestrial (soil) actinomycetes were the mainstay for antibiotic discovery efforts in the previous five decades, there is evidence that this resource is becoming exhausted. In contrast, the marine environment, covering more than three-fourths of the world's surface, represents a vast and relatively untapped source of novel scaffolds with unique antimicrobial properties. $^{7-10}$ Recently, structures identified from new marine actinomycete species have been shown to exhibit potent anti-MRSA activity with desirable cross-activity against other bacterial pathogens. ${ }^{11,12}$

In the course of screening a library of extracts from previously unidentified species of marine-derived actinomycetes for anti-MRSA activity, we followed a hit and purified an active secondary metabolite that we confirmed by NMR and mass spectrometric analysis to be etamycin-a classical depsipeptide antibiotic of the streptogramin B class. As decades have elapsed since consideration of the antibiotic properties of etamycin, we undertook an evaluation of its activity

\footnotetext{
${ }^{1}$ Skaggs School of Pharmacy and Pharmaceutical Sciences, University of California-San Diego, La Jolla, CA, USA; ${ }^{2}$ Center for Marine Biotechnology and Biomedicine, Scripps Institution of Oceanography, University of California-San Diego, La Jolla, CA, USA and ${ }^{3}$ Department of Pediatrics, University of California-San Diego, La Jolla, CA, USA ${ }^{4}$ Current address: Department of Chemistry, Harvey Mudd College, Claremont, CA 91711, USA. Correspondence: Dr ME Hensler, Department of Pediatrics, University of California, San Diego, 9500 Gilman Drive, MC 0687, La Jolla, CA 92093-0687, USA. E-mail: mhensler@ucsd.edu

Received 13 January 2010; revised 19 February 2010; accepted 22 February 2010; published online 26 March 2010
} 
against a panel of contemporary, clinically relevant HA- and CAMRSA strains. We find that etamycin exhibits favorable in vitro antibacterial activity and killing kinetics and is also protective against MRSA-induced lethality in a murine systemic infection model. These data suggest that etamycin could serve as the basis for in-depth study of related marine-derived molecules as MRSA-targeted therapies.

\section{MATERIALS AND METHODS}

\section{Bacterial strains}

A panel of HA- and CA-MRSA and other Gram-positive and -negative bacterial strains was used to probe the antimicrobial activity of etamycin. These included MRSA strains ATCC 33591, NRS22 (USA600), NRS71 (Sanger 252, genome strain), NRS119, USA200 (UAMS-1), NRS192, USA300 (UAMS-1182) and strain NRS386 (USA700). Isolates with the NRS designation were obtained through the Network of Antimicrobial Resistance in Staphylococcus aureus (NARSA) program supported under NIAID/NIH contract \# HHSN272200700055C. The USA200 and USA300 isolates were kind gifts of Greg Somerville at the University of Nebraska, Lincoln, and originally obtained from Mark Smeltzer at the University of Arkansas Medical Center. Other Gram-positive strains tested were Streptococcus pyogenes serotype M1 strain $5448,{ }^{13}$ Streptococcus agalactiae strain $\mathrm{COH} 1^{14}$ and vancomycin-resistant Enterococcus faecalis (ATCC 51299). The Gram-negative strains used were Pseudomonas aeruginosa (ATCC 27853), Escherichia coli (ATCC 25922), Moraxella catarrhalis (ATCC 25238) and Haemophilus influenzae (Type b, ATCC 10211).

The actinomycete strain CNS-575 was isolated from a sediment sample collected from the Nasese shoreline, Viti Levu, Fiji, at low tide from a depth of $\approx 0.5 \mathrm{~m}$ (coordinates $18.09^{\prime} 04.0 \mathrm{~S}$ and $178.27^{\prime} 11.4 \mathrm{E}$ ). The $16 \mathrm{~S}$ ribosomal RNA gene sequence (accession number Bankit 1235828) places this strain within the genus Streptomyces.

\section{Fermentation and isolation of etamycin}

Culture conditions. A $2 \mathrm{ml}$ frozen stock of actinomycete strain CNS-575 was used to inoculate $25 \mathrm{ml} \mathrm{Al}$ medium ( $10 \mathrm{~g}$ starch, $4 \mathrm{~g}$ yeast extract, $2 \mathrm{~g}$ peptone in 11 seawater) in a $125 \mathrm{ml}$ Erlenmeyer flask and shaken at $27^{\circ} \mathrm{C}$. The 5-day-old seed culture was then used to inoculate a 11 culture in Albfe $+\mathrm{C}$ (A1 media described above, with the addition of $1 \mathrm{~g} \mathrm{CaCO}_{3}, 5 \mathrm{ml}$ of $2 \%$ (w/v) $\mathrm{KBr}$ stock solution and $5 \mathrm{ml}$ of $0.8 \%(\mathrm{w} / \mathrm{v}) \mathrm{Fe}_{2}\left(\mathrm{SO}_{4}\right) \cdot 4 \mathrm{H}_{2} \mathrm{O}$ stock solution). Lastly, $25 \mathrm{ml}$ aliquots of the 3-day-old 11 culture were used to inoculate each of 1811 cultures in 2.81 Fernbach flasks containing medium Albfe+C. Strain CNS-575 was allowed to grow with shaking for 7 days at $27^{\circ} \mathrm{C}$ before extraction.

Isolation. Column chromatography was carried out on silica gel (Selecto Scientific, Suwanee, GA, USA; particle size 63-200 $\mu \mathrm{m}$ ). HPLC was carried out on a Beckman System Gold liquid chromatograph (126) (Beckman-Coulter, Brea, CA, USA) equipped with a Shimadzu diode array detector (SPDM10AVP) (Shimadzu Corporation, Kyoto, Japan) and using an Ultracarb ODS (30) column (Phenomenex, Torrance, CA, USA; $250 \times 10.0 \mathrm{~mm}, 5 \mu \mathrm{m}$ particle size). A 401 culture of strain CNS-575 was extracted using XAD-7 resin and eluted with acetone $(2 \times)$. The acetone solvent was removed by evaporation, and the remaining water was extracted three times with ethyl acetate. The obtained extract $(1.83 \mathrm{~g})$ was fractionated on silica gel (step gradient from 100:1 to 5:1 dichloromethane: methanol eluent). Fractions with antibacterial activity were eluted with 50:1 and 20:1 dichloromethane: methanol. The active fractions $(487 \mathrm{mg}$ ) were pooled and subjected to HPLC separation (isocratic, $60 \%$ aqueous acetonitrile eluent, $3 \mathrm{ml} \mathrm{min}^{-1}$ flow rate) to give etamycin (108.9 mg) after $31 \mathrm{~min}$.

\section{Spectroscopic analysis of etamycin}

All NMR spectra were measured using a Varian Oxford AS500 spectrometer (Varian Inc., Palo Alto, CA, USA) (5 mm double-resonance inverse broadband probe) at 500 and $125 \mathrm{MHz}$ for ${ }^{1} \mathrm{H}$ and ${ }^{13} \mathrm{C} \mathrm{NMR}$, respectively. Offline processing was conducted using Mestre-C NMR Software (Mestrelab Research, La Coruña, Spain; http://www.mestrec.com). ${ }^{1} \mathrm{H}$ and ${ }^{13} \mathrm{C}$ chemical shifts were referenced with the $\mathrm{CDCl}_{3}$ solvent peaks at $\delta 7.26$ and $\delta 77.0$, respectively (Figure 1 and Table 1). The IR spectrum was recorded in $\mathrm{KBr}$ on a Nicolet IR100 FTIR spectrometer (Thermo, Waltham, MA, USA). The UV spectrum

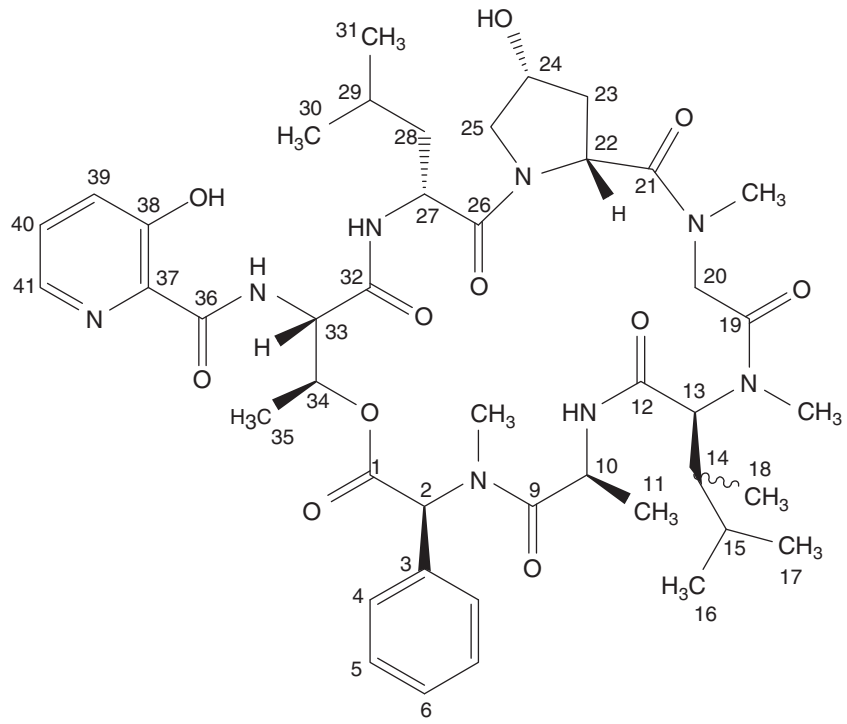

Figure 1 Chemical structure of etamycin. (Numbered atoms correspond to those in Table 1.).

was recorded in methanol on a Beckman Coulter DU800 spectrophotometer (Beckman-Coulter). The optical rotation was measured in methanol on a Jasco P-2000 polarimeter (Jasco, Inc., Easton, MD, USA). HR-MS were acquired at the UCSD Molecular Mass Spectrometry Facility.

\section{Susceptibility testing}

Minimum inhibitory concentration (MIC) assays were performed by microbroth dilution. Briefly, an overnight culture of the test strain was diluted to 1:100 in broth and grown to mid-logarithmic phase at $37^{\circ} \mathrm{C}$ in a shaking incubator. Todd-Hewitt broth (obtained from Hardy Diagnostics, Santa Maria, CA, USA) was used for all strains except $H$. influenzae, for which the susceptibility testing was carried out in brain-heart infusion media supplemented with hemin $\left(10 \mu \mathrm{g} \mathrm{ml}^{-1}\right)$ and $\mathrm{NAD}\left(10 \mu \mathrm{g} \mathrm{ml}^{-1}\right)$. The culture was pelleted and resuspended to $\mathrm{OD}_{600}=0.4$ in phosphate-buffered saline. The bacteria were then added to a 96-well test plate containing etamycin dilutions in broth; the final bacterial concentration was $5 \times 10^{5} \mathrm{CFU} \mathrm{ml}^{-1}$ in a final volume of $100 \mu$ l. Vancomycin (Hospira, Lake Forest, IL, USA) was used as a control for all MRSA strains. Other control antibiotics included: ampicillin (Sandoz, Princeton, NJ, USA) for E. faecalis and Streptococcus spp, cloramphenicol (Sigma Chemical, St Louis, MO, USA) for M. catarrhalis and H. influenzae, and ciprofloxacin (Bedford Laboratories, Bedford, OH, USA) for $P$. aeruginosa and E. coli. The test plate containing the etamycin dilutions and target bacteria was incubated at $37^{\circ} \mathrm{C}$ in a shaking incubator for $18-20 \mathrm{~h}$. MIC was determined to be the lowest concentration of etamycin that inhibited bacterial growth as determined by turbidometric assessment at $\mathrm{OD}_{600}$.

\section{Cytotoxicity assay}

Analysis of mammalian cell cytotoxicity was carried out essentially as described. ${ }^{15}$ Briefly, etamycin dilutions were added to HeLa cells $\left(2 \times 10^{4}\right.$ cells per well) in a sterile 96-well plate. Cytotoxicity was assayed by MTS at $24 \mathrm{~h}$ using the CellTiter 96 AQueous non-radioactive cell proliferation assay according to the manufacturer's instructions (Promega, Madison, WI, USA). Plates were read at $A_{490}$ in a Versamax microplate reader (Molecular Devices, Mountain View, CA, USA).

\section{In vitro time-kill assays}

Time-kill assays were carried out as previously described ${ }^{16-18}$ with modifications. Briefly, MRSA strain USA300 (UAMS-1182) was cultured overnight at $37^{\circ} \mathrm{C}$ in Todd-Hewitt broth. The bacteria were then grown to mid-logarithmic phase and prepared in phosphate-buffered saline as for the MIC assays. Test tubes were prepared in duplicate containing vehicle control, etamycin, or 
Table $1{ }^{1} \mathrm{H}$ NMR, ${ }^{13} \mathrm{C}$ NMR and ${ }^{15} \mathrm{~N}$ NMR of etamycin (each rotamer) in $\mathrm{CDCl}_{3}$

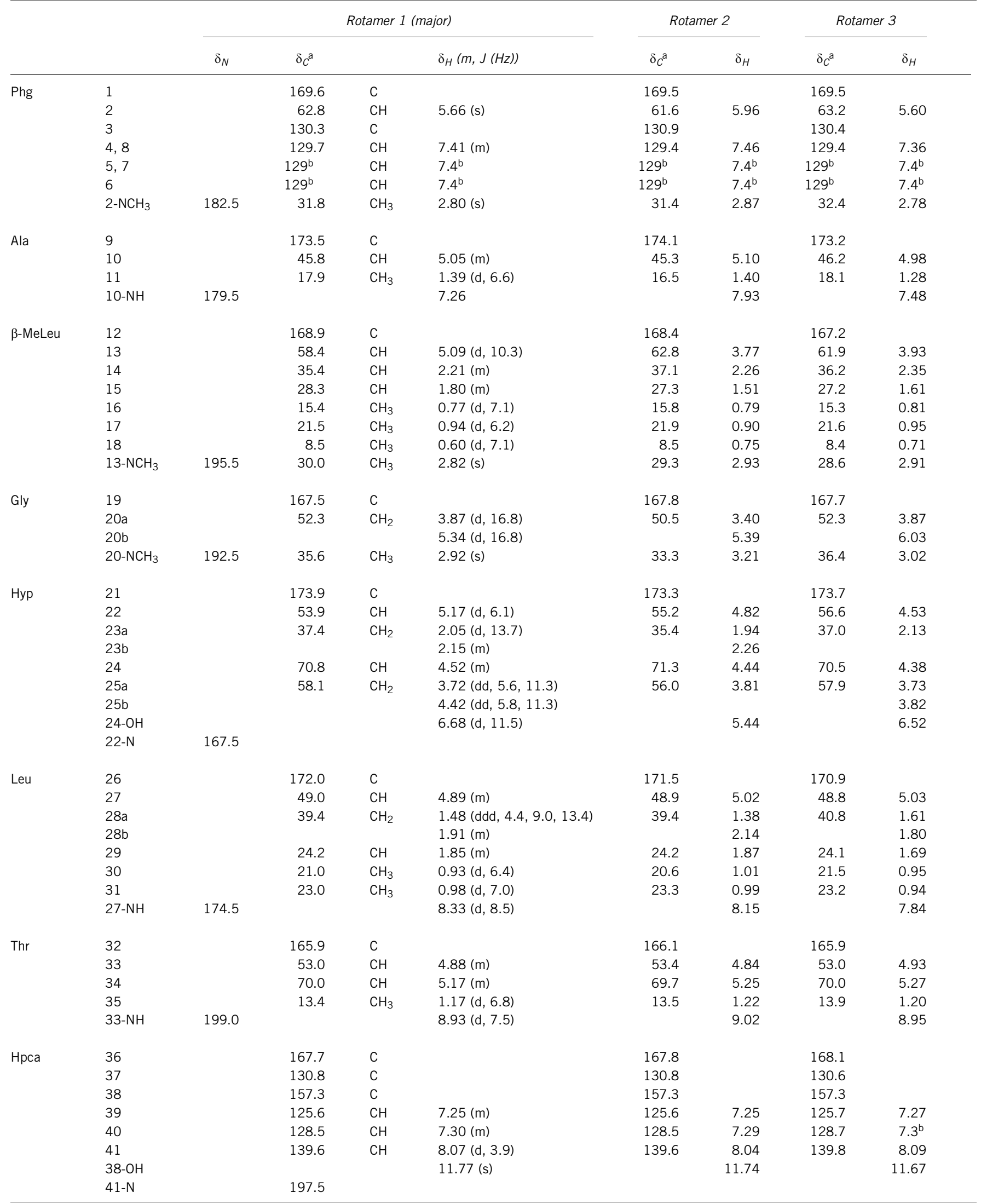

\footnotetext{
${ }^{13} \mathrm{C}$ shifts obtained from HMBC and HSQC spectra.
} 
vancomycin at concentrations of 1,4 or 10 times the MIC of $4 \mu \mathrm{g} \mathrm{ml}^{-1}$ and $1.5 \mu \mathrm{g} \mathrm{ml}^{-1}$, respectively. Bacteria were added to each tube at a starting inoculum of $\sim 5 \times 10^{5} \mathrm{CFU} \mathrm{ml}^{-1}$ in a final volume of $5 \mathrm{ml}$. The cultures were incubated in a $37^{\circ} \mathrm{C}$ shaking incubator, and aliquots were removed from each test tube at $0,3,6$ and $24 \mathrm{~h}$. These aliquots were serially diluted in phosphatebuffered saline and plated on Todd-Hewitt agar plates in the absence of antibiotics. Colonies were enumerated after $24 \mathrm{~h}$ at $37^{\circ} \mathrm{C}$. The limit of detection of this assay equated to $40 \mathrm{CFU} \mathrm{ml}^{-1}$.

\section{In vivo studies}

Female CD1 mice ( 8 weeks old) (Charles River, Wilmington, MA, USA) were injected i.p. with $2 \times 10^{9} \mathrm{CFU}$ of HA-MRSA strain Sanger 252 in $4 \%$ mucin. At 1 and $8 \mathrm{~h}$ after infection, the mice were injected i.p. with etamycin $\left(20 \mathrm{mg} \mathrm{kg}^{-1}\right)$ or an equivalent volume of vehicle control. The mice were observed for signs of sepsis, including lethargy, piloerection and dehydration. Survival was monitored for a total of $72 \mathrm{~h}$, and mice that became moribund during the study were humanely euthanized, as were any surviving mice at the end of the study. These studies were reviewed and approved by the University of California San Diego Animal Subjects Committee.

\section{RESULTS}

The actinomycete strain designated CNS-575 was cultured from a marine sediment collected along the coast of Fiji. The 16S rRNA gene sequence analysis places this strain within the genus Streptomyces; however, it shares $<98 \%$ sequence identity with any formally described species, suggesting that it may represent a new species. Solid-phase extraction and chromatographic purification yielded an 879 molecular weight peptide, which appeared as three distinct rotamers in the NMR spectrum. The presence of rotamers was not affected by changes of NMR solvent or acquisition temperature, and we ultimately performed the NMR-based structure elucidation of all three rotamers simultaneously (Table 1 ). The resulting planar structure was consistent with that of the previously reported terrestrially derived streptogramin, etamycin ${ }^{19,20}$ (Figure 1), although a complete chemical shift assignment of etamycin has not previously been reported. Acid hydrolysis followed by analysis using advanced Marfey's method ${ }^{21}$ revealed the stereochemical configuration to be consistent with etamycin (see Supplementary Information for a detailed discussion of the Marfey's analysis).

Etamycin was originally isolated from terrestrial actinomycete $S$. griseus alongside the streptogramin-A antibiotic griseoviridin, and the two molecules together displayed synergism common for other streptogramins, including bactericidal activity primarily against some Gram-positive organisms. ${ }^{22}$ We investigated in more detail the antibiotic properties of etamycin as a single agent against a panel of clinically relevant MRSA strains and discovered it to possess very good activity. MIC ranged from $1-2 \mathrm{mgl}^{-1}$ for CA-MRSA strains and certain HA-MRSA strains, to $8-16 \mathrm{mgl}^{-1}$ for the HA-MRSA strains ATCC 33591 and Sanger 252 (Table 2). Etamycin also showed a low level of activity against strains of the Gram-positive bacteria $S$. pyogenes and $S$. agalactiae $\left(\mathrm{MIC}=8 \mathrm{mgl}^{-1}\right.$ ) and minimal activity against vancomycin-resistant $E$. faecalis $\left(\mathrm{MIC}=16 \mathrm{mgl}^{-1}\right)$. The spectrum of etamycin activity extended to strains of the Gram-negative coccobacilli and respiratory tract pathogens $M$. catarrhalis $\left(\mathrm{MIC}=1 \mathrm{mgl}^{-1}\right)$ and to a lesser extent $H$. influenzae $\left(\mathrm{MIC}=16 \mathrm{mgl}^{-1}\right)$, but not to other Gram-negative pathogens tested including E. coli, S. typhimurium and P. aeruginosa. To assess a potential therapeutic index for etamycin against MRSA, we analyzed its effects in a mammalian HeLa cell cytotoxicity assay. We found that etamycin exhibited no cytoxicity at concentrations up to $128 \mathrm{mgl}^{-1}$ (data not shown), suggesting the potential for a favorable therapeutic index against MRSA.
Table 2 Antibacterial activity of etamycin

\begin{tabular}{lc} 
Strain & $M I C\left(\mathrm{mg}^{-1}\right)$ \\
\hline HA- and CA-MRSA strains & \\
UAMS-1 (USA200) & $1-2$ \\
Sanger 252 & $8-16(2)^{\mathrm{a}}$ \\
ATCC 33591 & 16 \\
NRS22 (USA600) & $1-4$ \\
NRS119 (linezolid-resistant strain) & $1-2$ \\
UAMS-1182 (USA300) & 4 \\
NRS192 & 2 \\
NRS386 (USA700) & 2 \\
& \\
Non-MRSA strains & \\
E. faecalis (vanB) & $16(16)$ \\
S. pyogenes (M1 strain 5448) & 8 \\
S. agalactiae (serotype III strain COH1) & 8 \\
M. catarrhalis (ATCC 25238) & 1 \\
H. influenzae (type b, ATCC 10211) & 16 \\
E. coli (ATCC 25922) & $>256$ \\
S. typhimurium (ATCC 13311) & $>256$ \\
P. aeruginosa (ATCC 27853) & $>256$
\end{tabular}

Abbreviations: CA, community associated; HA, hospital associated; MIC, minimum inhibitory concentrations; MRSA, methicillin-resistant Staphylococcus aureus.

aValues in parentheses represent MICs for Synercid against these bacterial strains tested under identical conditions as for etamycin.

bStrain NRS386 is associated with both community and health-care infections.

We studied the time-kill kinetics of etamycin at different concentrations for comparison against vancomycin, the most common agent used in current MRSA therapy. Evaluation was conducted at $1 \times, 4 \times$ and $10 \times$ the respective MIC of each drug against the CA-MRSA (USA300) strain UAMS-1182 (Figure 2a). As shown in Figure 2b, etamycin killed UAMS-1182 somewhat more rapidly than vancomycin at $10 \times$ MIC during the first $6 \mathrm{~h}$; however, vancomycin was able to reduce the number of viable bacteria to below the threshold of detection of this assay by $24 \mathrm{~h}$, whereas killing by etamycin, although substantial, remained incomplete. Collectively, these data suggest that etamycin acts in both time- and concentration-dependent mechanisms and is faster-acting but significantly less bactericidal than vancomycin.

As a first assessment of the in vivo efficacy of etamycin, we used a murine systemic infection model, conservatively choosing as the challenge strain MRSA Sanger 252, against which etamycin exhibited a higher MIC of $8-16 \mathrm{mgl}^{-1}$. Female CD1 mice ( 8 weeks old) were treated with etamycin $\left(20 \mathrm{mg} \mathrm{kg}^{-1}\right)$ or vehicle control at 1 and $8 \mathrm{~h}$ after i.p. MRSA infection, and general health and survival were followed up for up to $72 \mathrm{~h}$. Within $6-12 \mathrm{~h}$ of MRSA infection, most mice in the control group exhibited signs of sepsis including lethargy, dehydration, and piloerection, whereas the severity of these symptoms was greatly reduced or absent in most of the etamycin-treated animals. As shown in Figure 3, the two doses of etamycin offered significant protection to mice infected with MRSA during the $72 \mathrm{~h}$ monitoring period ( $20 \%$ mortality vs $75 \%$ morality in controls).

\section{DISCUSSION}

For the first time from a marine-derived actinomycete we isolated the streptogramin, etamycin, and found that it had marked antibacterial activity against clinically important strains of MRSA. Although etamycin was first identified more than five decades ago, its antibiotic properties had not been characterized in detail. Those early studies also describe etamycin as a bacteriostatic compound against the 

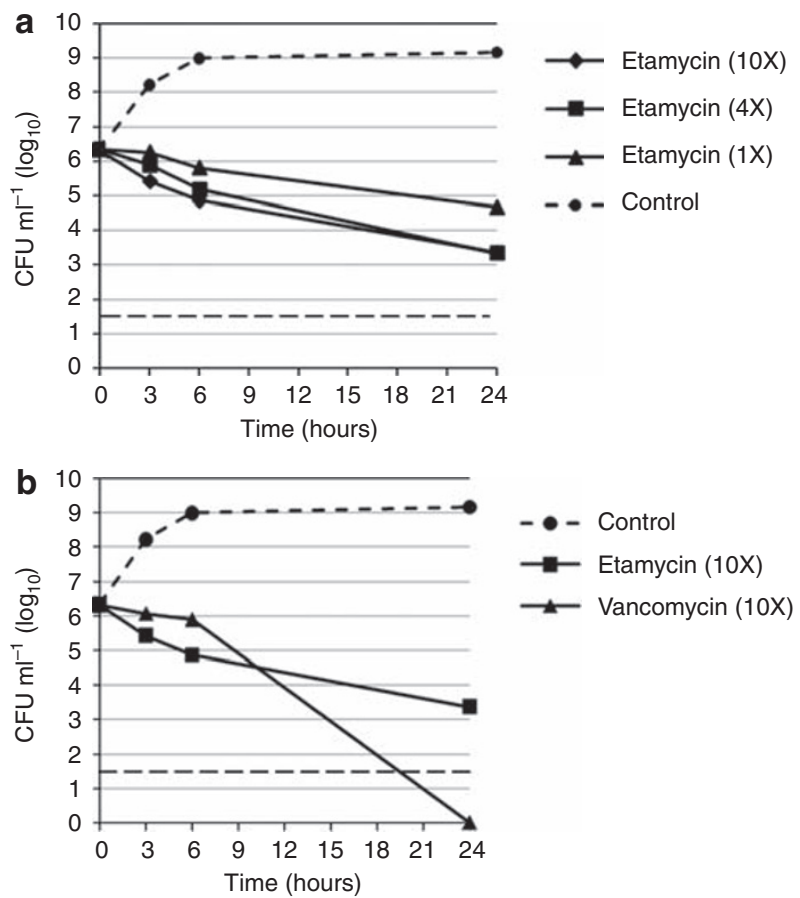

Figure 2 In vitro time-kill kinetics of etamycin against CA-MRSA strain UAMS1182. (a) Concentration dependence of etamycin at one, four and ten times the MIC over $24 \mathrm{~h}$. MRSA was incubated with the specified concentrations of etamycin, and surviving bacteria were enumerated at the given times by quantitative culture on agar in the absence of the compound. Data represent the mean of three independent experiments, and the dotted line at $1.6 \log _{10}$ represents the limit of detection of the assay. (b) Comparison of killing kinetics of etamycin with vancomycin at ten times the MIC for each drug. Surviving bacteria were enumerated at the given times by quantitative culture on agar. The data represent the mean of three independent experiments, and the dotted line at $1.6 \log _{10}$ represents the limit of detection of the assay.

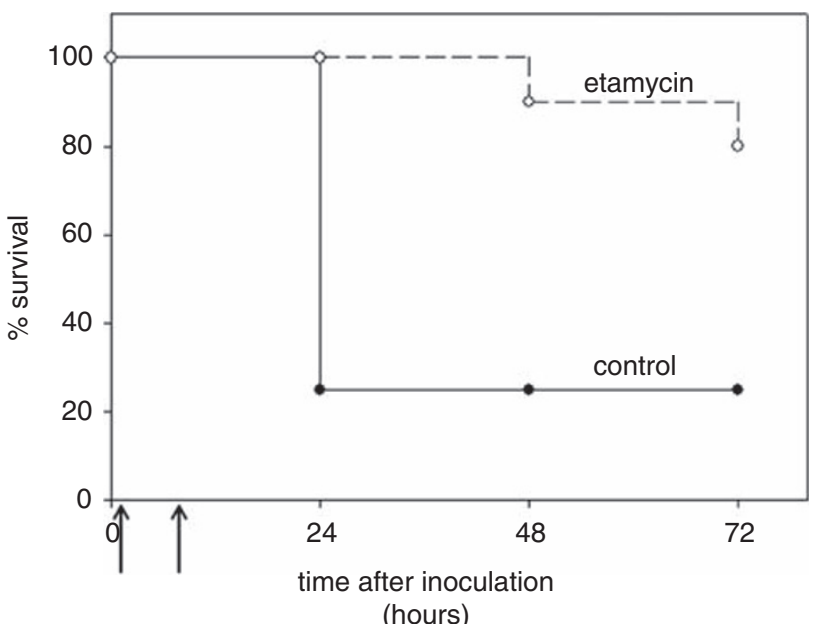

Figure 3 In vivo efficacy of etamycin in a murine model of MRSA sepsis. Female CD1 mice ( 8 week old) were injected i.p. with $2 \times 10^{9} \mathrm{CFU}$ of $\mathrm{HA}$ MRSA strain Sanger 252 in $1 \%$ mucin. At 1 and $8 \mathrm{~h}$ after infection (indicated by arrows), the mice were injected i.p. with either $20 \mathrm{mg} \mathrm{kg}^{-1}$ etamycin $(n=10)$ or DMSO vehicle control $(n=8)$. The mice were monitored for signs of sepsis for up to $72 \mathrm{~h}$, and mice that became moribund were humanely euthanized.
S. aureus strain Duncan. ${ }^{22}$ We found that etamycin alone at high concentration may exhibit limited bactericidal activity against contemporary CA-MRSA strains, as after $24 \mathrm{~h}$ of exposure to etamycin at $4 \times$ MIC, $<1 \%$ of the starting inoculum of CA-MRSA strain UAMS1182 remained viable (Figure 2), representing an approximate three-log kill by $24 \mathrm{~h}$. Furthermore, the progressive decrease in the number of surviving bacteria over time even at $1 \times$ MIC also supports a conclusion that etamycin exhibits some, although certainly incomplete, bactericidal activity over $24 \mathrm{~h}$. However, despite this result, etamycin clearly does not kill CA-MRSA as effectively as vancomycin in the 24-h assay.

The streptogramin antibiotic, quinupristin-dalfopristin (Synercid) is currently approved for use in complicated cutaneous infections caused by multiple drug-resistant Gram-positive cocci, including MRSA and vancomycin-resistant Enterococcus faecium. ${ }^{23}$ In this combination, streptogramins A and B together act to inhibit protein synthesis, with quinupristin (A) preventing polypeptide elongation by binding to the $50 \mathrm{~S}$ ribosomal subunit and dalfopristin (B) binding to another site on the $50 \mathrm{~S}$ ribosomal subunit and inducing a conformational change enhancing quinupristin activity. ${ }^{24}$ In our assays we found that etamycin, presumably through protein synthesis inhibition, exhibits significant activity against MRSA alone without the benefit of its streptogramin A partner. Etamycin also demonstrated a very low level of activity against $E$. faecalis $\left(\mathrm{MIC}=16 \mathrm{mgl}^{-1}\right.$ ), a frequently drug-resistant nosocomial pathogen that is associated with urinary tract and surgical wound infections, ${ }^{25}$ and this activity seemed comparable to the very low level of activity of Synercid against this pathogen in our assay. Clearly, if etamycin were ever considered for introduction into clinical use, defined breakpoints would need to be determined.

Our isolation of etamycin from a novel marine-derived actinomycete species emphasizes the utility of the marine environment as a source of antibacterials, ${ }^{7}$ in particular marine-derived actinomycetes that have been shown to naturally produce a wide variety of active parent molecules and analogs. ${ }^{6,11,26}$ Etamycin was previously isolated from the terrestrial microbe, $S$. griseoviridus, naturally occurring as multiple congeners in which a hydroxyproline residue was replaced by a proline. ${ }^{27}$ In addition, limited structure-activity relationship data point to the importance of the picolinyl moiety of etamycin for its activity. ${ }^{19}$ Future studies will further investigate the activity of etamycin analogs from marine-derived actinomycetes for characterization and optimization of activity toward further preclinical development.

\section{ACKNOWLEDGEMENTS}

This work was supported by the National Institutes of Health Training Program in Marine Biotechnology (T32 GM067550 to NH), by the National Institutes of Health (RO1GM084350-01 to WF and VN), by the National Institutes of Health International Cooperative Biodiversity Groups program (U01-

TW007401-01 to WF and PRJ), and by the National Institutes of Health Cancer Therapeutics Training Program (T32 CA121938 to KNM). We gratefully acknowledge R Raju and B Aalbersberg for providing the actinomycete strain CNS-575. We also acknowledge George Sakoulas, MD, for many helpful discussions.

1 Cornaglia, G. \& Rossolini, G. M. Forthcoming therapeutic perspectives for infections due to multidrug-resistant Gram-positive pathogens. Clin. Microbiol. Infect. 15, 218223 (2009).

2 Como-Sabetti, K et al. Community-associated methicillin-resistant Staphylococcus aureus: trends in case and isolate characteristics from six years of prospective surveillance. Public Health Rep. 124, 427-435 (2009). 
3 Patel, M. Community-associated meticillin-resistant Staphylococcus aureus infections: epidemiology, recognition and management. Drugs 69, 693-716 (2009).

4 Boucher, H. W. et al. Bad bugs, no drugs: no ESKAPE! An update from the Infectious Diseases Society of America. Clin. Infect. Dis. 48, 1-12 (2009).

5 Spellberg, B. et al. The epidemic of antibiotic-resistant infections: a call to action for the medical community from the Infectious Diseases Society of America. Clin. Infect. Dis. 46, 155-164 (2008).

6 Demain, A. L. Antibiotics: natural products essential to human health. Med. Res. Rev. 29, 821-842 (2009).

7 Fenical, W. \& Jensen, P. R. Developing a new resource for drug discovery: marine actinomycete bacteria. Nat. Chem. Biol. 2, 666-673 (2006).

8 Kwon, H. C., Kauffman, C. A., Jensen, P. R. \& Fenical, W. Marinomycins A-D, antitumor-antibiotics of a new structure class from a marine actinomycete of the recently discovered genus 'marinispora'. J. Am. Chem. Soc. 128, 1622-1632 (2006).

9 Mincer, T. J., Jensen, P. R., Kauffman, C. A. \& Fenical, W. Widespread and persistent populations of a major new marine actinomycete taxon in ocean sediments. Appl. Environ. Microbiol. 68, 5005-5011 (2002).

10 Soria-Mercado, I. E., Prieto-Davo, A., Jensen, P. R. \& Fenical, W. Antibiotic terpenoid chloro-dihydroquinones from a new marine actinomycete. J. Nat. Prod. 68, 904-910 (2005).

11 Hughes, C. C., Prieto-Davo, A., Jensen, P. R. \& Fenical, W. The marinopyrroles, antibiotics of an unprecedented structure class from a marine Streptomyces sp. Org Lett. 10, 629-631 (2008).

12 McArthur, K. A. et al. Lynamicins A-E, chlorinated bisindole pyrrole antibiotics from a novel marine actinomycete. J. Nat. Prod. 71, 1732-1737 (2008).

13 Datta, V. et al. Mutational analysis of the group A streptococcal operon encoding streptolysin S and its virulence role in invasive infection. Mol. Microbiol. 56, 681-695 (2005).

14 Doran, K. S., Chang, J. C., Benoit, V. M., Eckmann, L. \& Nizet, V. Group B streptococcal beta-hemolysin/cytolysin promotes invasion of human lung epithelial cells and the release of interleukin-8. J. Infect. Dis. 185, 196-203 (2002).
15 Jere, D. et al. Poly (amino ester) composed of poly (ethylene glycol) and aminosilane prepared by combinatorial chemistry as a gene carrier. Pharm. Res. 25, 875-885 (2008).

16 Ueda, Y. et al. In vitro and in vivo antibacterial activities of SM-216601, a new broadspectrum parenteral carbapenem. Antimicrob. Agents Chemother. 49, 4185-4196 (2005).

17 Credito, K., Lin, G. \& Appelbaum, P. C. Activity of daptomycin alone and in combination with rifampin and gentamicin against Staphylococcus aureus assessed by time-kill methodology. Antimicrob. Agents Chemother. 51, 1504-1507 (2007).

18 Tsuji, B. T., Yang, J. C., Forrest, A., Kelchlin, P. A. \& Smith, P. F. In vitro pharmacodynamics of novel rifamycin ABI-0043 against Staphylococcus aureus. J. Antimicrob. Chemother. 62, 156-160 (2008)

19 Bateman, K. P., Thibault, P., Yang, K., White, R. L. \& Vining, L. C. Probing the substrate specificity of an enzyme catalyzing inactivation of streptogramin B antibiotics using LCMS and LC-MS/MS. J. Mass Spectrom. 32, 1057-1063 (1997).

20 Sheehan, J. C. \& Ledis, S. L. Total synthesis of a monocyclic peptide lactone antibiotic, etamycin. J. Am. Chem. Soc. 95, 875-879 (1973).

21 Fujii, K., Ikai, Y., Oka, H., Suzuki, M. \& Harada, K. A. Nonempirical method using LC/ MS for determination of the absolute configuration of constituent amino acids in a peptide: combination of Marfey's method with mass spectrometry and its practical application. Anal. Chem. 69, 5146-5151 (1997).

22 Garcia-Mendoza, C. Studies on the mode of action of etamycin (Viridogrisein). Biochim. Biophys. Acta. 97, 394-396 (1965).

23 Metzger, R., Bonatti, H. \& Sawyer, R. Future trends in the treatment of serious grampositive infections. Drugs Today (Barc) 45, 33-45 (2009).

24 Allington, D. R. \& Rivey, M. P. Quinupristin/dalfopristin: a therapeutic review. Clin. Ther. 23, 24-44 (2001).

25 Sood, S., Malhotra, M., Das, B. K. \& Kapil, A. Enterococcal infections \& antimicrobial resistance. Indian J. Med. Res. 128, 111-121 (2008).

26 Demain, A. L. \& Sanchez, S. Microbial drug discovery: 80 years of progress. J Antibiot (Tokyo) 62, 5-16 (2009)

27 Chopra, C. et al. Congeners of etamycin produced by Streptomyces griseoviridus. J. Antibiot. (Tokyo) 32, 392-401 (1979).

Supplementary Information accompanies the paper on The Journal of Antibiotics website (http://www.nature.com/ja) 